\title{
Localización Biométrica de los Puntos Motores del Músculo Cuádriceps Femoral de Individuos Brasileños
}

\author{
Biometric Localization of the Motor Points of the \\ Femoral Quadriceps Muscle of Brazilian Individuals
}

\author{
Alveal-Mellado, D. ${ }^{1}$; Sousa-Rodrigues, C. F. ${ }^{2}$ \& Olave, E. ${ }^{1,3}$
}

ALVEAL-MELLADO, D. ; SOUSA-RODRIGUES, C. F. \& OLAVE, E. Localización biométrica de los puntos motores del músculo cuádriceps femoral de individuos brasileños. Int. J. Morphol., 37(4):1498-1503, 2019.

RESUMEN: El músculo cuadríceps femoral es un músculo que participa principalmente en los movimientos de la rodilla y también en la cadera, pudiendo ser afectado por alteraciones en el trofismo muscular, tras lesiones de estas articulaciones o afecciones en el tono muscular, como la espasticidad, tras lesiones cerebrovasculares. Cada una de sus cabezas está inervada por ramos del nervio femoral. El presente estudio tuvo por objetivo la identificación y medición de la distancia de los puntos motores (Pm) del músculo cuadríceps femoral, en relación a estructuras anatómicas de relevancia clínica. Se utilizaron 30 miembros inferiores de 23 cadáveres formolizados, de individuos brasileños adultos. El estudio fue realizado en la Universidade Estadual de Ciências da Saúde de Alagoas (UNCISAL), Maceió, Brasil. Se realizó la disección por planos en la zona medial, anterior y lateral del muslo, identificando a los ramos del nervio femoral para cada componente, músculos recto femoral ( $\mathrm{mRf})$, vasto medial $(\mathrm{mVm})$, vasto intermedio ( $\mathrm{mVint})$ y vasto lateral (mVlat), localizando cada uno de los Pm. Las mediciones se realizaron con un cáliper marca Mitutoyo de 0,05 mm de precisión. Los datos obtenidos fueron tratados estadísticamente, utilizando para ello, el software STATA (versión 14.1). El mRf, tuvo un promedio de $2,45 \pm 1,39 \mathrm{Pm}$, estando la gran mayoría de los Pm (63\%), en la zona distal del tercio proximal del muslo; el mVm presentó 4,42 $\pm 2,74$ Pm en promedio, encontrándose el $41 \%$ de ellos en el tercio medio del muslo, zona distal; el mVint tuvo 3,99 $\pm 2,34$ Pm en promedio y el $58 \%$ de ellos, se localizaron en la zona proximal del tercio medio del muslo; el mVlat presentó un promedio de 3,88 $\pm 2,37$ Pm y el 50 $\%$ de éstos se encontraron en del tercio proximal del muslo. La localización biométrica de los Pm se informa en resultados. La ubicación biométrica de los Pm, favorecerá el quehacer, tanto clínico como quirúrgico, de la zona anterior del muslo.

PALABRAS CLAVE: Anatomía; Músculo cuádriceps femoral; Inervación; Puntos motores.

\section{INTRODUCCIÓN}

El músculo cuádriceps femoral (mCf) se ubica en la zona anterior del muslo y está constituido por el músculo vasto medial ( $\mathrm{mVm}$ ), el músculo vasto intermedio (mVint), el músculo vasto lateral (mVlat) y el músculo recto femoral (mRf). Las fibras de los cuatro músculos, se originan en diferentes estructuras óseas de la pelvis y el fémur, insertándose distalmente alrededor de la patela (Standring, 2016).

Diferentes autores han propuesto que el $\mathrm{mVm}$ se compone a su vez de 2 porciones, más o menos definidas, denominadas porción larga y porción oblicua (Jojima et al., 2004; Peeler et al., 2005; Rajput et al., 2017). Así mismo, se ha publicado también que el mVlat presenta las porciones superficial, intermedia y profunda (Toia et al., 2015; D'Arpa et al., 2016). Adicionalmente, se ha señalado que el mVint presenta subdivisiones que podrían considerarse un componente extra del mCf, constituyendo así, un quinto músculo, denominado tensor del vasto intermedio (Grob et al., 2016).

Debido a su volumen, longitud y la orientación de sus fibras, el mCf participa en movimientos de la articulación de la cadera, y principalmente de la rodilla. También es frecuentemente afectado por alteraciones en el trofismo muscular, tras lesiones de estas articulaciones, o afecciones en el tono muscular, como la espasticidad, tras lesiones cerebrovasculares.

Por otra parte, el punto motor (Pm) de un músculo, representa el lugar donde el ramo motor del nervio, ingresa al vientre muscular, penetrando su fascia (Sung et al.,

\footnotetext{
${ }^{1}$ Programa de Magister en Ciencias, mención Morfología, Universidad de La Frontera, Temuco, Chile.

${ }^{2}$ Universidade de Ciências da Saude de Alagoas, Maceió, Brasil

${ }^{3}$ Facultad de Medicina, Universidad de La Frontera, Temuco, Chile.
} 
2003). El conocimiento de la ubicación detallada de los Pm de un músculo, permite mejorar la efectividad de procedimientos como la estimulación eléctrica (Botter et al., 2011), que favorece la recuperación del trofismo muscular. Además, facilita la identificación del punto de inyección de anestésicos y anticolinérgicos, para disminuir la espasticidad (Albert et al., 2002).

El presente estudio se enfoca en la identificación y medición de la distancia de los Pm del mCf, en relación a estructuras anatómicas de relevancia clínica. Así mismo, relaciona la ubicación de éstos puntos, con la longitud del muslo.

\section{MATERIAL Y MÉTODO}

Se utilizaron 30 miembros inferiores, 13 derechas y 17 izquierdas, de 23 cadáveres de individuos brasileños adultos, conservados en solución de formaldehído al $10 \%$. El estudio se llevó a cabo en dependencias de la Universidade Estadual de Ciências da Saúde de Alagoas (UNCISAL), en la ciudad de Maceió, Brasil.

La disección comenzó con la remoción de la inserción del ligamento inguinal, en la espina ilíaca anterosuperior (EIAS), el cual se reflejó hacia medial, para identificar al $\mathrm{NF}$, a nivel de la base del triángulo femoral. Se realizó la disección por planos en la zona medial, anterior y lateral del muslo. Tras separar las estructuras adiposas y fasciales, se prescindió de los elementos vasculares y se realizó el seguimiento de los ramos que emite el NF para el mRf; mVm; mVint y mVlat, hasta identificar cada uno de los Pm.

Tras esto, se procedió a medir la longitud del muslo de cada muestra, establecida como la distancia comprendida entre una línea horizontal que pasa por la EIAS, denominada "Eje x" y otra línea horizontal que pasa por los puntos más prominentes de los epicóndilos femorales, medial y lateral, denominada línea biepicondilar (LBE).

Para localizar con mayor precisión los Pm, la distancia Eje x-LBE se dividió en tercios proximal, medio y distal, los cuales se dividieron una vez más, en dos partes; zona proximal y distal.

La localización biométrica de los Pm, se determinó según la distancia vertical al "Eje x" y en relación a la distancia horizontal, a una línea vertical, paralela al muslo, que se origina en la EIAS, denominada "Eje y" (Fig. 1). Las mediciones se realizaron utilizando un cáliper marca Mitutoyo de 0,05 $\mathrm{mm}$ de precisión.

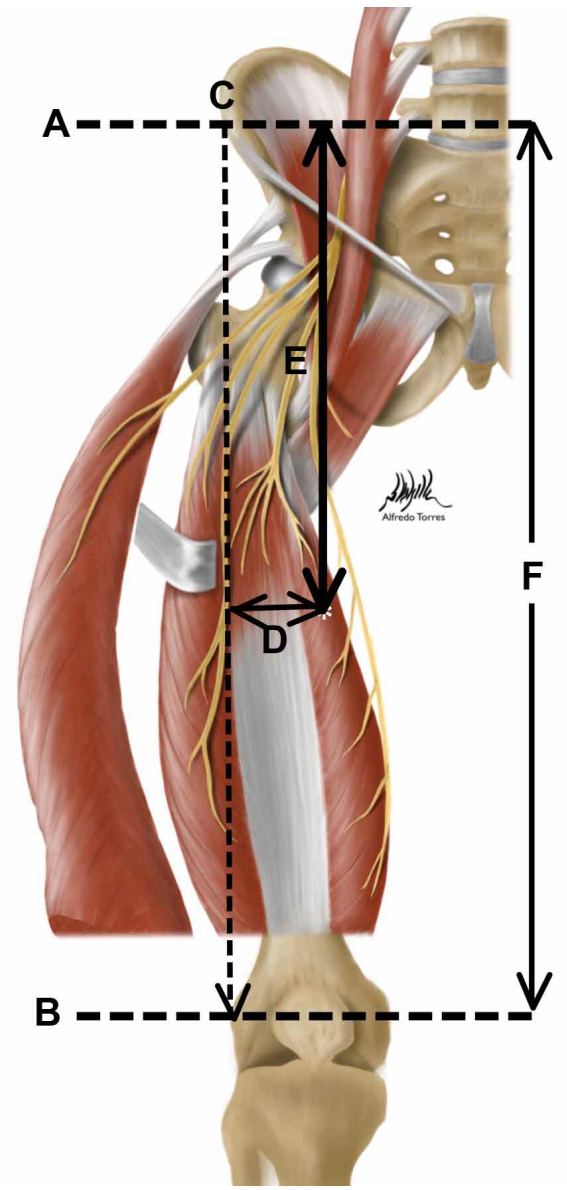

Fig.1. Método de medición y localización de los puntos motores (Pm) del músculo cuádriceps femoral (mCf). A: "Eje x", que pasa por la espina ilíaca anterosuperior (EIAS). B: Línea biepicondilar (LBE) femoral. C :"Eje y", que se origina en la EIAS y se dirige verticalmente, paralelo al muslo, en dirección distal. D: Distancia horizontal del punto motor (Pm) al "Eje y". E: Distancia vertical del Pm al "Eje x". F: Longitud del muslo. Asterisco blanco: Pm.

Los datos obtenidos fueron tratados estadísticamente, comparando los resultados entre cada uno de los grupos, formados por los componentes del mCf. Para ello, se utilizó el software estadístico STATA (versión 14.1).

Se utilizó la prueba estadística de Kruskal-Wallis, para comparar el promedio de Pm, distancia al "Eje x" y distancia al "Eje y" entre cada uno de los músculos.

\section{RESULTADOS}

El análisis del mRf, reveló la existencia de 2,45 \pm $1,39 \mathrm{Pm}$ en promedio. Ubicándose éstos a una distancia vertical de 136,26 \pm 38,36 mm al "Eje x". En relación al 
“Eje y", los Pm se localizaron a una distancia horizontal de $10,87 \pm 15,77 \mathrm{~mm}$, en dirección medial. En cuanto a su distribución en el muslo, la gran mayoría de los Pm (63\%), se concentraron en la zona distal (Zd) del tercio proximal (Tp).

El mVm presentó 4,42 2 2,74 Pm en promedio. Se localizaron éstos a 245,76 \pm 67,50 mm del "Eje x" y a 43,82 $\pm 12,18 \mathrm{~mm}$ hacia medial del "Eje y". El $41 \%$ se encontró

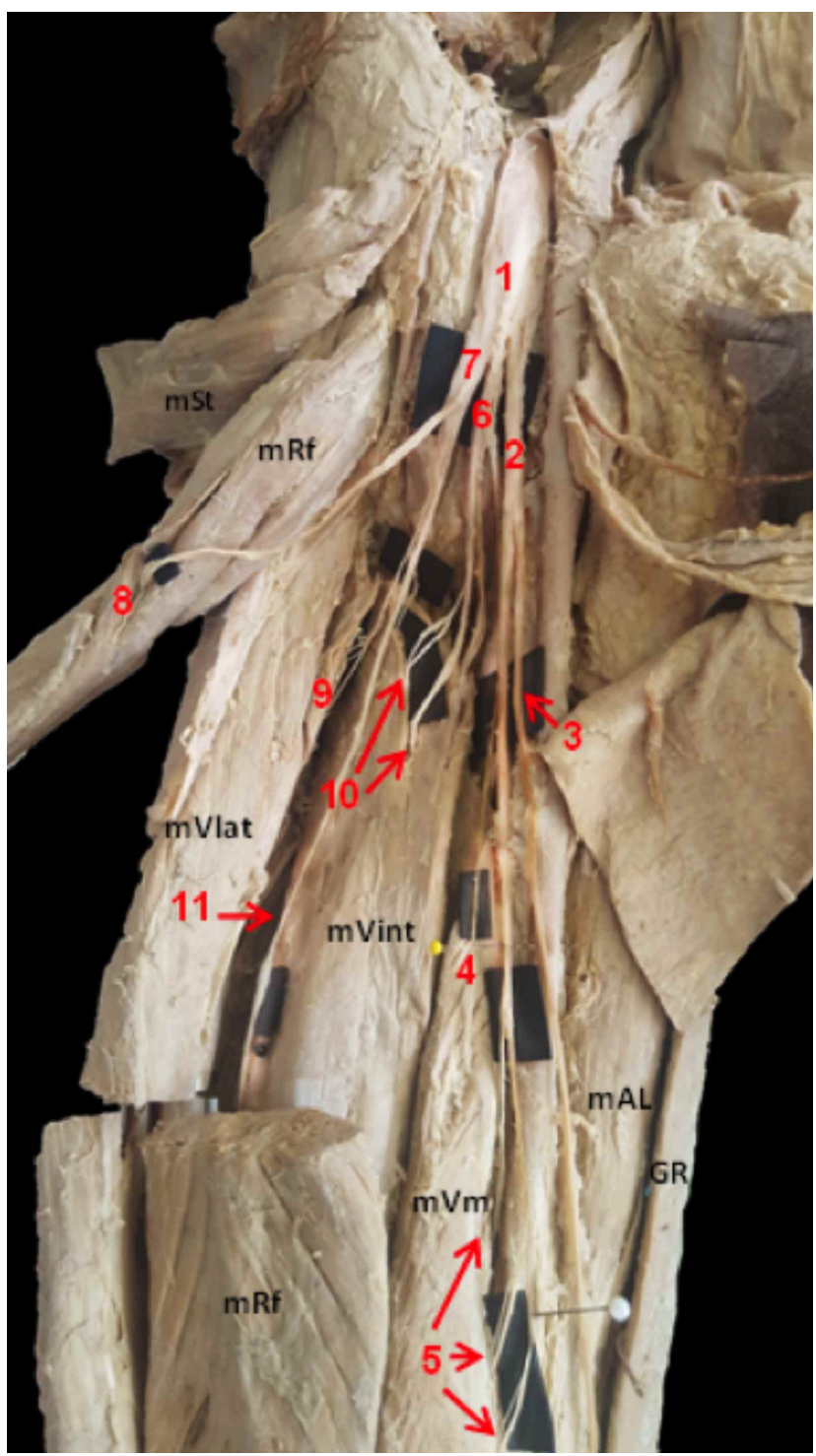

Fig. 2. Distribución del NF en el mCf. 1: Nervio Femoral, 2: Ramo hacia el $\mathrm{mVm}, 3$ : Nervio Safeno, 4: Punto motor de la porción superior (larga) del $\mathrm{mVm}, 5$ : Puntos motores de la porción inferior (oblicua) del mVm, 6: Ramo hacia el mVint, 7, Ramo hacia el mRf y mVlat, 8: Puntos motores del mRf, 9:Puntos motores del mVlat, 10: Puntos motores del mVint, 11: Ramo hacia zona distal del mVint, mSt: Músculo Sartorio, mRf: Músculo Recto femoral, mVlat: Músculo vasto lateral, mVint: Músculo vasto intermedio, mVm: Músculo vasto medial, mAL: Músculo aductor largo, GR: Músculo grácil. en el tercio medio (Tm) del muslo, específicamente en la zona distal (Zd) (Fig. 2).

Al mVint le correspondieron 3,99 $\pm 2,34$ Pm en promedio. Los cuales se localizaron a 169,45 \pm 39,68 mm del "Eje x" y a 7,53 \pm 15,79 mm hacia medial del "Eje y". El 58 $\%$ de ellos se ubicaron en la zona proximal ( $\mathrm{Zp}$ ) del tercio medio (Tm) del muslo (Figs. 2 y 3).

Finalmente el mVlat presentó 3,88 \pm 2,37 Pm en promedio. Éstos se localizaron a 163,70 $\pm 50,46 \mathrm{~mm}$ del “Eje x" y a 9,76 \pm 15,18 mm lateralmente al "Eje y". El 50 $\%$ de los Pm se encontraron en la zona distal (Zd) del tercio proximal (Tp) del muslo (Fig. 3).

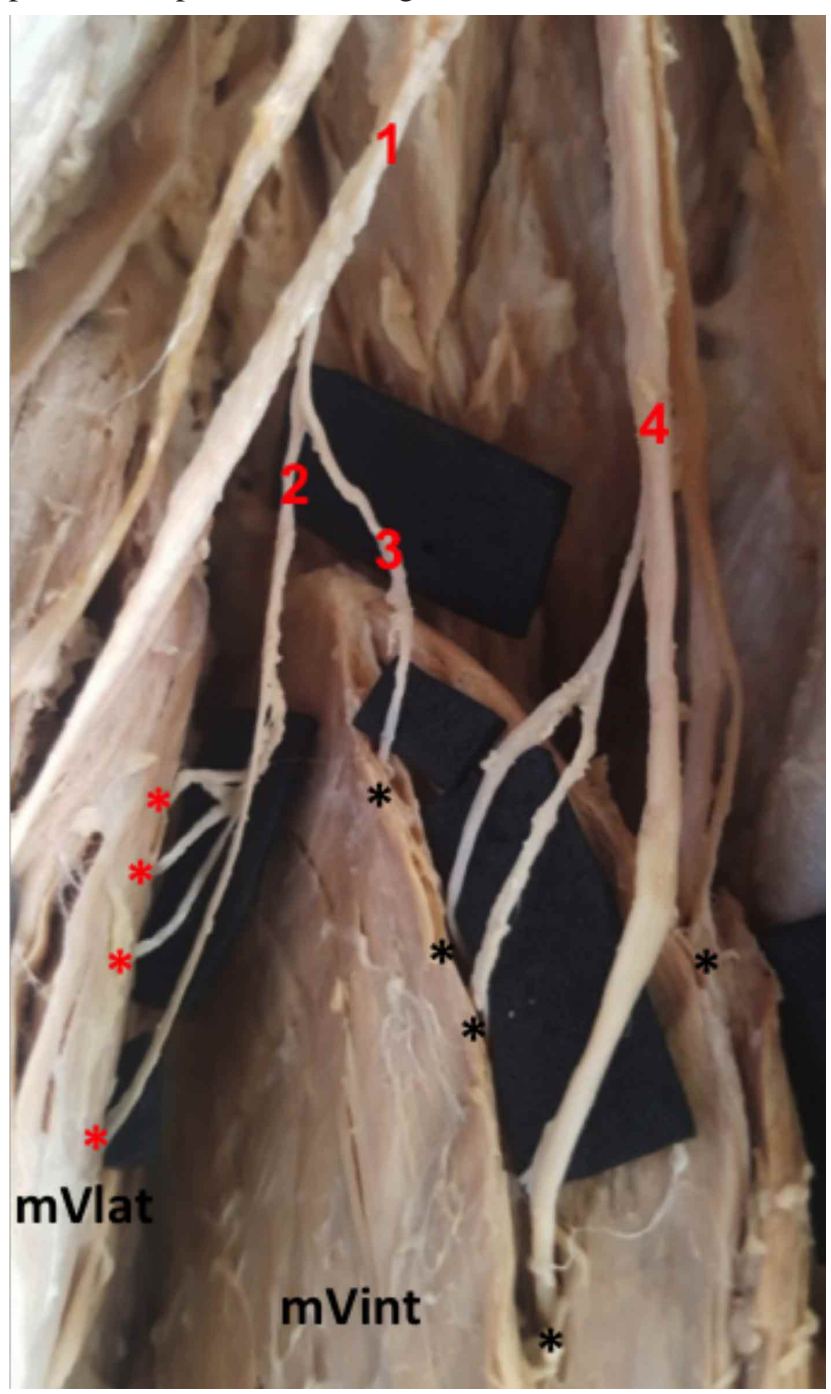

Fig. 3. Imagen con acercamiento de los puntos motores del mVlat y mVint. 1: Ramo primario del NF dirigido al mVlat, 2: Ramo secundario del NF dirigido al mVlat, 3: Ramo terciario del NF dirigido al mVint. 4: Ramo primario del NF dirigido al mVint, Asteriscos rojos: Puntos motores del mVlat, Asteriscos negros: Puntos motores del mVint. 


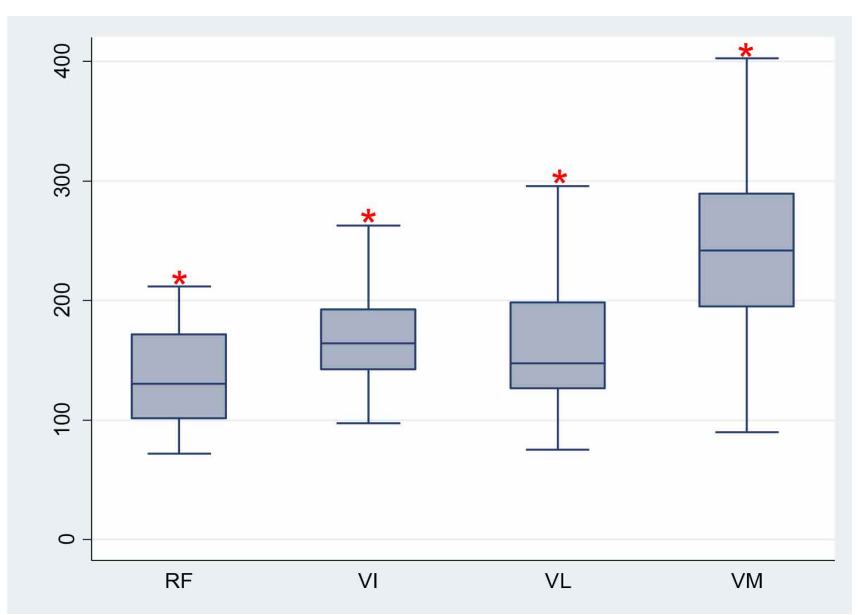

Fig. 4. Gráfico de cajas de la distancia vertical, de los Pm de cada músculo al "Eje x". La caja está delimitada por el intervalo de valores comprendidos entre los percentiles $25-75 \%$. La mediana está representada por la línea divisoria. Los bigotes representan los valores extremos. Asterisco rojo: $\mathrm{p}<0,05$.

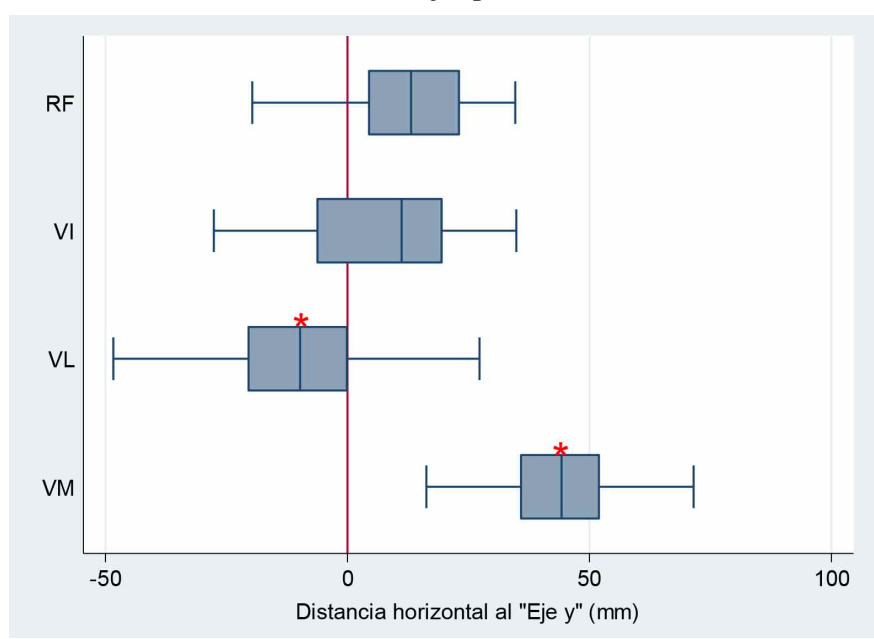

En la Tabla I se muestran las distancias promedio de los Pm en cada músculo, en relación a los puntos de referencia anatómicos. La Tabla II muestra la localización de los Pm según las zonas en que se dividió el muslo.

En relación a la cantidad de Pm, sólo existió diferencia estadísticamente significativa entre el mRf y los demás componentes del $\mathrm{mCf}$, el cual presentó la menor cantidad de ellos.

Al comparar las distancias verticales de los $\mathrm{Pm}$ al "Eje x", entre los cuatro músculos del mCf, hubo diferencias estadísticamente significativas $(p<0,05)$ en todos ellos (Fig. 4).

Al realizar la comparación de las distancias horizontales al "Eje y" de los Pm, entre los cuatro músculos, solamente hubo diferencias estadísticamente significativas $(\mathrm{p}<0,05)$ para el $\mathrm{mVlat}$ y $\mathrm{mVm}$. No existieron diferencias estadísticas entre el mRf y mVint (p>0,05) (Fig. 5).

Fig. 5. Gráfico de cajas de la distancia horizontal de los Pm de cada músculo al "Eje y". La caja está delimitada por el intervalo de valores comprendidos entre los percentiles $25-75 \%$. La mediana está representada por la línea divisoria. Los bigotes representan los valores extremos. Valores negativos representan la ubicación lateral al "Eje y". Valores positivos representan la ubicación medial al "Eje y", Asterisco rojo: p<0,05.

Tabla I. Promedio de las distancias de los Pm a los Ejes X e Y.

\begin{tabular}{lcccccc}
\hline Músculos & $\begin{array}{c}\mathrm{N} \\
\text { Muestras }\end{array}$ & $\begin{array}{c}\text { Media Pm } \\
(\mathrm{DE})\end{array}$ & $\begin{array}{c}\text { Distancia vertical } \\
\text { en mm a "Eje x" } \\
(\mathrm{DE})\end{array}$ & Rango (mm) & $\begin{array}{c}\text { Distancia } \\
\text { horizontal en mm } \\
\text { a “Eje y" (DE) }\end{array}$ & Rango (mm) \\
\hline mRf & 15 & $2,45(1,39)$ & $136,26(38,36)$ & 72,09 a 211,66 & $10,87(15,77)$ & $-36,44$ a 34,67 \\
mVm & 30 & $4,4(2,74)$ & $245,76(67,50)$ & 89,83 a 402,56 & $43,82(12,18)$ & 16,33 a 71,56 \\
mVint & 30 & $3,9(2,34)$ & $169,45(39,68)$ & 97,46 a 317,21 & $7,53(15,79)$ & $-27,68$ a 34,85 \\
mVlat & 30 & $3,88(2,37)$ & $163,70(50,46)$ & 75,49 a 295,53 & $9,76(15,18)$ & $-48,46$ a 27,22 \\
\hline
\end{tabular}

Tabla II. Localización de los Pm del mCf en los tercios y zonas del muslo.

\begin{tabular}{|c|c|c|c|c|c|c|c|}
\hline \multirow[t]{2}{*}{ Músculos } & \multicolumn{2}{|c|}{$\begin{array}{c}\text { TP } \\
\mathbf{N}^{\circ} \text { de } \operatorname{casos}(\%)\end{array}$} & \multicolumn{2}{|c|}{ 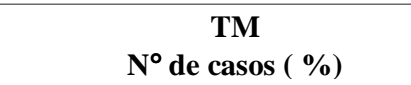 } & \multicolumn{2}{|c|}{$\begin{array}{c}\text { TD } \\
N^{\circ} \text { de } \operatorname{casos}(\%)\end{array}$} & \multirow[t]{2}{*}{ Total } \\
\hline & $\mathbf{Z p}$ & Zd & $\mathbf{Z p}$ & Zd & $\mathbf{Z p}$ & Zd & \\
\hline$\overline{\mathrm{mRf}}$ & $1(2,0 \%)$ & $32(62,7 \%)$ & $18(35,3 \%)$ & & & & 51 \\
\hline $\mathrm{mVm}$ & & $14(6,9 \%)$ & $70(34,3 \%)$ & $83(40,7 \%)$ & $35(17,2 \%)$ & $2(1,0 \%)$ & 204 \\
\hline mVint & & $67(35,6 \%)$ & $109(58,0 \%)$ & $11(5,9 \%)$ & $1(0,5 \%)$ & & 188 \\
\hline mVlat & $1(0,58 \%)$ & $87(50,3 \%)$ & $58(33,5 \%)$ & $26(15,0 \%)$ & $1(0,6 \%)$ & & 173 \\
\hline
\end{tabular}

$\mathrm{TP}=$ tercio proximal $\mathrm{TM}=$ tercio medio $; \mathrm{tD}=\mathrm{Tercio}$ distal $\mathrm{Zp}=$ zona proximal $\mathrm{Zd}=$ zona distal. 


\section{DISCUSIÓN}

En cuanto a la cantidad de Pm del mCF y su ubicación dentro de las porciones del muslo, Reid (1920) reportó los hallazgos tras realizar la disección de un cadáver. En la muestra, tanto el mRf como el mVint presentaron cuatro Pm cada uno, al mVlat correspondieron cinco Pm y el $\mathrm{mVm}$ presentó seis $\mathrm{Pm}$. Si bien es cierto, en el presente estudio, ninguna de las muestras analizadas, presentó tal disposición, existe similitud en cuanto el $\mathrm{mVm}$ fue quien presentó la mayor cantidad de Pm. En relación a su distribución, Reid describe que los Pm del mRf y mVlat se ubicaron en la mitad proximal y tercio medio del muslo, respectivamente, para el $\mathrm{mVm} \mathrm{y}$ mVint, se localizaron en el tercio medio. Lo cual es similar a lo encontrado en nuestro estudio.

Más recientemente Lefebvre et al. (2006), estudiaron la inervación del $\mathrm{mCf}$ a través de estimulación eléctrica en 8 sujetos sanos. Los resultados revelaron la existencia de un $\mathrm{Pm}$, tanto para el mRf como para el mVlat y tres Pm para el $\mathrm{mVm}$. Aunque no reportaron la cantidad de Pm para el mVint, ni la localización de los Pm en relación a puntos de referencia anatómicos.

También a través de estimulación eléctrica, Botter et al. localizaron dos $\mathrm{Pm}$, tanto para el $\mathrm{mRf}$, como para el $\mathrm{mVm}$ y tres Pm para el mVlat. Los Pm del mRf y mVlat, se ubicaron en el tercio medio del muslo, y al igual que en nuestro estudio, el $\mathrm{mVm}$ presentó la ubicación más distal de Pm, en el tercio distal. Los autores no reportaron información referente al mVint, posiblemente debido a su ubicación, en un plano muscular más profundo, difícil de abarcar con electroestimulación.

Varios estudios, se han centrado en la identificación de los ramos del NF que inervan al mRf. Tal es el caso de Koshima et al. (1994), quienes basándose en la disección de cuatro muslos, establecieron que "el ramo motor del NF hacia el mRf se subdivide en varios ramos, antes de alcanzar el tercio proximal del músculo". Por otro lado, Spyriounis (2006), tras disecar diez muslos, manifestaron que "el ramo del NF dirigido al mRf, emitió de 2 a 4 ramos que penetraron el músculo en su tercio proximal". Sung et $a l$., en su análisis de veintidós cadáveres, reportaron la existencia de $2 \mathrm{Pm}$ para el $\mathrm{mRf}$. Misma cantidad que fue presentada por Page et al. (2019), quienes estudiaron dieciséis muslos de 8 cadáveres.

Descripciones aisladas de la cantidad de Pm, de los demás componentes del mCf, han sido reportadas por Peeler et al., quienes reportan que "el ramo del NF nunca ingresó al vientre del mVm en más de un solo sitio". Mientras que Rozen et al. (2009) se centraron en el mVlat y manifestaron que "de 4 a 7 ramos principales se distribuyeron en el músculo".

Nuestro estudio considera a la EIAS y la línea biepicondilar del fémur, como los puntos anatómicos de referencia, a partir del cual se realizaron las mediciones. Los Pm para el mRf se localizaron más próximos a la EIAS y los correspondientes al $\mathrm{mVm}$ fueron los más distales. Hallazgos similares a los presentados por Page et al., quienes utilizaron como puntos de referencia el sitio de emergencia del NF en el ligamento inguinal y el margen superior de la patela.

Tayfur et al. (2010) estudiaron la anatomía del mVlat en 30 cadáveres. Encontrando una distancia promedio de 194,6 mm (145-213 mm) desde la EIAS hasta el Pm. Punto de localización más distal a lo reportado en el presente estudio (163,70 $\pm 50,46 \mathrm{~mm}$ ) (Tabla I).

El $\mathrm{mVm}$ fue quien presentó la mayor variabilidad en la localización de los Pm, según su distancia a la EIAS (Fig. 4), lo que concuerda con las descripciones de Jojima et al., Lefebvre et al. y Rajput et al., quienes establecen la inervación del músculo mediante ramos cortos del NF, para la porción proximal, y ramos largos para su porción distal, tal como se muestra en la Figura 2.

Además, en nuestro estudio se determinó la distancia horizontal de cada Pm, a una línea de referencia vertical, "Eje $\mathrm{y}$ ". De esta manera, los Pm pertenecientes al $\mathrm{mVm}$ se encontraron en el rango de valores más mediales, y los pertenecientes al mVlat a los más laterales (Fig. 5). Por otro lado, no existió diferencia estadísticamente significativa entre el promedio de la distancia horizontal de los Pm del mRf y mVint a este eje. Lo que se explica debido a la ubicación de ambos músculos, en un mismo eje vertical, estando el primero inmediatamente delante del segundo.

En relación a esta coordenada horizontal, Albert et al. (2000) localizaron el punto motor principal para el mVint, a $20 \pm 5 \mathrm{~mm}$ laterales a la línea vertical, que une el punto de salida del NF a nivel del ligamento inguinal y el polo superior de la patela. Línea de referencia distinta a la utilizada en nuestro estudio, donde el promedio de Pm para este músculo se ubicó hacia medial del "Eje y".

Finalmente, podemos afirmar que el nuestro, es de los primeros estudios que se enfoca en realizar una descripción de la cantidad de Pm, de cada uno de los constituyentes del mCf, basándose en el análisis de un tamaño muestral considerable. La literatura reporta que, de las porciones que conforman mCf, el mRf presenta la menor cantidad de Pm. Tanto el $\mathrm{mVm}$, como mVlat, presentan alta variabilidad en número y el mVint, es el menos estudiado de los cuatro. 
Nuevos estudios, que consideren la ubicación de los Pm, según su distancia a puntos de referencia anatómicos, favorecerá el quehacer, tanto clínico como quirúrgico, de la zona anterior del muslo.

\section{AGRADECIMIENTOS}

Al Sr. Damian Chandía-Poblete por su colaboración en el análisis estadístico, al ilustrador Sr. Alfredo Torres por su aporte en la esquematización de figuras y por últimos, a la familia de todas las personas fallecidas, que donaron sus cuerpos, para llevar a cabo el estudio anatómico.

ALVEAL-MELLADO, D. ; SOUSA-RODRIGUES, C. F. \& OLAVE, E. Biometric localization of the motor points of the femoral quadriceps muscle of brazilian individuals. Int. J. Morphol., 37(4):1498-1503, 2019

SUMMARY: The quadriceps femoris muscle is a muscle that participates mainly in the movements of the knee and also in the hip, being able to be affected by alterations in muscle trophism, after injuries of these joints or muscular tone conditions, such as spasticity, after injuries cerebrovascular. Each of its heads is innervated by branches of the femoral nerve. The objective of this study was to identify and measure the distance of the motor points (MP) of the quadriceps femoris muscle, in relation to anatomical structures of clinical relevance. Thirty lower limbs of 23 formolized corpses of adult Brazilian individuals were used. The study was conducted at the State University of Ciências da Saúde de Alagoas (UNCISAL), Maceió, Brazil. The dissection was performed by planes in the medial, anterior and lateral thigh, identifying the femoral nerve branches for each component, rectus femoris muscles $(\mathrm{Rfm})$, medial vastus $(\mathrm{mVm})$, vastus intermedius (int $\mathrm{Vm}$ ) and vastus lateralis ( lat $\mathrm{Vm}$ ), locating each of the MP. The measurements were made with a Mitutoyo caliper of 0.05 mm accuracy. The data obtained were treated statistically, using the STATA software (version 14.1). The Rfm had an average of $2.45 \pm 1.39 \mathrm{MP}$, the great majority of the MP $(63 \%)$ being in the distal area of the proximal third of the thigh; the $\mathrm{mVm}$ presented $4.42 \pm 2.74 \mathrm{MP}$ on average, with $41 \%$ of them in the middle third of the thigh, distal zone; the intVm had 3.99 $+2.34 \mathrm{MP}$ on average and $58 \%$ of them were located in the proximal area of the middle third of the thigh; the latVm presented an average of $3.88 \pm 2.37 \mathrm{MP}$ and $50 \%$ of these were found in the proximal third of the thigh. The biometric localization of the MP is reported in results. The biometric location of the MP, will favor the task, both clinical and surgical of the anterior thigh area.

KEY WORDS: Anatomy; Femoral quadriceps muscle; Innervation; Motor points.

\section{REFERENCIAS BIBLIOGRÁFICAS}

Albert, T. A.; Yelnik, A.; Bonan, I.; Lebreton, F. \& Bussel, B. Effectiveness of femoral nerve selective block in patients with spasticity: preliminary results. Arch. Phys. Med. Rehabil., 83(5):692-6, 2002.

Albert, T.; Yelnik, A.; Colle, F.; Bonan, I. \& Lassau, J. P. Anatomic motor point localization for partial quadriceps block in spasticity. Arch. Phys. Med. Rehabil., 81(3):285-7, 2000.

Botter, A.; Oprandi, G.; Lanfranco, F.; Allasia, S.; Maffiuletti, N. A. \& Minetto, M. A. Atlas of the muscle motor points for the lower limb: implications for electrical stimulation procedures and electrode positioning. Eur. J. Appl. Physiol., 111(10):2461-71, 2011.

D’Arpa, S.; Toia, F.; Brenner, E.; Melloni, C.; Moschella, F. \& Cordova, A. Variability and reliability of the vastus lateralis muscle anatomy. Acta Chir. Belg., 116(4):203-12, 2016.

Grob, K.; Ackland, T.; Kuster, M. S.; Manestar, M. \& Filgueira, L. A newly discovered muscle: The tensor of the vastus intermedius. Clin. Anat., 29(2):256-63, 2016.

Jojima, H.; Whiteside, L. A. \& Ogata, K. Anatomic consideration of nerve supply to the vastus medialis in knee surgery. Clin. Orthop. Relat. Res., (423):15760, 2004.

Koshima, I.; Moriguchi, T.; Soeda, S.; Hamanaka, T.; Tanaka, H. \& Ohta, S. Free rectus femoris muscle transfer for one-stage reconstruction of established facial paralysis. Plast. Reconstr. Surg., 94(3):421-30, 1994.

Lefebvre, R.; Leroux, A.; Poumarat, G.; Galtier, B.; Guillot, M.; Vanneuville, G. \& Boucher, J. P. Vastus medialis: anatomical and functional considerations and implications based upon human and cadaveric studies. J. Manipulative Physiol. Ther., 29(2):139-44, 2006.

Page, B. J.; Mrowczynski, O. D.; Payne, R. A.; Tilden, S. E.; Lopez, H.; Rizk, E. \& Harbaugh, K. The relative location of the major femoral nerve motor branches in the thigh. Cureus, 11(1):e3882, 2019.

Peeler, J.; Cooper, J.; Porter, M. M.; Thliveris, J. A. \& Anderson, J. E. Structural parameters of the vastus medialis muscle. Clin. Anat., 18(4):281-9, 2005.

Rajput, H. B.; Rajani, S. J. \& Vaniya, V. H. Variation in morphometry of vastus medialis muscle. J. Clin. Diagn. Res., 11(9):AC01-AC04, 2017.

Reid, R. W. Motor points in relation to the surface of the body. J. Anat., 54(Pt. 4):271-5, 1920.

Rozen, W. M.; le Roux, C. M.; Ashton, M. W. \& Grinsell, D. The unfavorable anatomy of vastus lateralis motor nerves: a cause of donor-site morbidity after anterolateral thigh flap harvest. Plast. Reconstr. Surg., 123(5):15059, 2009

Spyriounis, P. K. The extended approach to the vascular pedicle of the anterolateral thigh perforator flap: anatomical and clinical study. Plast. Reconstr. Surg., 117(3):997-1001, 2006.

Standring, S. Gray's Anatomy: The Anatomical Basis of Clinical Practice. $41^{\text {st }}$ ed. New York, Elsevier, 2016.

Sung, D. H.; Jung, J. Y.; Kim, H. D.; Ha, B. J. \& Ko, Y. J. Motor branch of the rectus femoris: anatomic location for selective motor branch block in stifflegged gait. Arch. Phys. Med. Rehabil., 84(7):1028-31, 2003.

Tayfur, V.; Magden, O.; Edizer, M. \& Atabey, A. Anatomy of vastus lateralis muscle flap. J. Craniofac. Surg., 21(6):1951-3, 2010.

Toia, F.; D'Arpa, S.; Brenner, E.; Melloni, C.; Moschella, F. \& Cordova, A. Segmental anatomy of the vastus lateralis: guidelines for muscle-sparing flap harvest. Plast. Reconstr. Surg., 135(1):185e-98e, 2015.

Dirección para correspondencia:

Dr. Enrique Olave

Facultad de Medicina

Universidad de La Frontera

Av. Francisco Salazar 01145, Temuco

CHILE

Recibido : 26-05-2019

Aceptado: 19-06-2019

Email: enrique.olave@ufrontera.cl 